\title{
Electromagnetic Compatibility Studies: LTE BS vs. Aeronautical Radionavigation Services in 694-790 MHz Frequency Band
}

\author{
Evaldas Stankevičius (Doctoral Student, Vilnius Gediminas Technical University), \\ Šarūnas Oberauskas (Deputy Head of Spectrum Engineering Division, \\ Communications Regulatory Authority of the Republic of Lithuania)
}

\begin{abstract}
This paper presents the sharing analysis of the 694-790 MHz frequency band for Mobile services IMT and Aeronautical radio-navigation services (ARNS) using SEAMCAT (established by CEPT) software based on the statistical simulation (Monte-Carlo) method. In 2012 the World Radiocommunication Conference (WRC-12) decided to allocate the $694-790 \mathrm{MHz}$ frequency band (the so-called $700 \mathrm{MHz}$ band) to mobile services IMT (excluding aeronautical mobile) after WRC-15 conference. But this agreement raises electromagnetic compatibility problems, which should be solved until WRC-15 [1]. This study was carried out in two phases: first applying theoretical analysis, then statistical Monte-Carlo simulations with SEAMCAT software tool in order to verify results obtained in theoretical approach. Analytical calculations shows that the required protection distances between ARNS stations and the MS base stations are $132 \mathrm{~km}$. The obtained results from SEAMCAT simulations indicate that separation distance should be above $100 \mathrm{~km}$. These results illustrate that the systems are not electromagnetically compatible. The possible mitigation technic could be antenna pattern correction.
\end{abstract}

Keywords - Aircraft navigation; Base stations; Electromagnetic compatibility; Interchannel interference; Radio spectrum management.

\section{INTRODUCTION}

The beginning of Mobile Services IMT networks establishment in $694 \mathrm{MHz}-790 \mathrm{MHz}$ is after the World Radiocommunication Conference in 2015 (WRC-15). This agreement concerns only administrations from ITU (International Telecommunication Union) Region 1 - Europe, Russia, the Middle East and Africa. This frequency range can be put into use only when all the necessary electromagnetic compatibility studies are completed. These compatibility studies should be performed by CEPT and ITU organizations and concluded by 2015 . The neighbouring countries have to find possible solutions for the coordination procedures of IMT systems in $694 \mathrm{MHz}-790 \mathrm{MHz}$ band. [1].

The following generic case study elaborates on the possible interference from the Mobile Services to the ARNS Service in the $694 \mathrm{MHz}-790 \mathrm{MHz}$ frequency band.

Assuming that the provisions provided in the GE-06 Agreement [14] apply, it is shown that the deployment of mobile service in a duel situation to the ARNS ground receivers is not possible in addition to already existing deployment of digital terrestrial television (DTT) transmitters. Furthermore, the study assumes coexistence between mobile and broadcasting service might be unlikely in co-channel situation due to the large required separation distances between the stations of the services [3].

Noting the required separation distances between stations of ARNS and broadcasting service, the related co-channel interference respectively and the lesser probability of interferences from mobile service base stations into ARNS ground receivers at much shorter distances, a combination of both impacts to the ARNS ground receivers can be neglected [3].

In order to reduce required separation distances the first step would be to limit emission power levels of base/mobile stations. In this frequency band will operate UMTS or LTE technologies, which have the ability to efficiently manage the radiated power to ensure the acceptable level of quality. The studies show that the power management efficiency of 3GPP technologies can be increased by using cognitive control mechanism [4]. The radiated power can be reduced by changing the mobile network concept, which moving from the macro to femto or micro cell formation [5] and [6].

Other mitigation techniques can also be used in this case, e.g. antenna beam forming, precise mobile network planning taking into account the location of ARNS stations, interfering antenna azimuth angles, mechanical and electrical tilts control and etc. [7] and [8].

\section{GENERAL TECHNICAL CHARACTERISTICS FOR MONTE-CARLO SIMULATIONS}

All assumptions were derived from Annex 2 and Annex 6 of the JTG Chairman's Report [2] and Recommendation ITU-R M.1830 [9].

The Recommendation ITU-R M.1830 provides characteristics of ARNS systems. The extract of basic parameters for the $694 \mathrm{MHz}-790 \mathrm{MHz}$ frequency band, is presented in Table I.

TABLE I

BASIC CHARACTERISTICS OF THE ARNS SYSTEM OPERATING IN THE $694 \mathrm{MHZ}-790 \mathrm{MHZ}$ FREQUENCY BAND

\begin{tabular}{|l|l|l|l|}
\hline $\begin{array}{l}\text { ARNS system } \\
\text { type }\end{array}$ & $\begin{array}{l}\text { Operating } \\
\text { frequencies, } \\
\text { MHz }\end{array}$ & $\begin{array}{l}\text { Bandwidth, } \\
\text { MHz }\end{array}$ & $\begin{array}{l}\text { Receiver antenna } \\
\text { height, } \mathbf{m}\end{array}$ \\
\hline RSBN & $\begin{array}{l}772,776,780, \\
784,788\end{array}$ & 3 or 0.7 & 10 \\
\hline RLS 2 & 740 & 8 & 10 \\
\hline
\end{tabular}




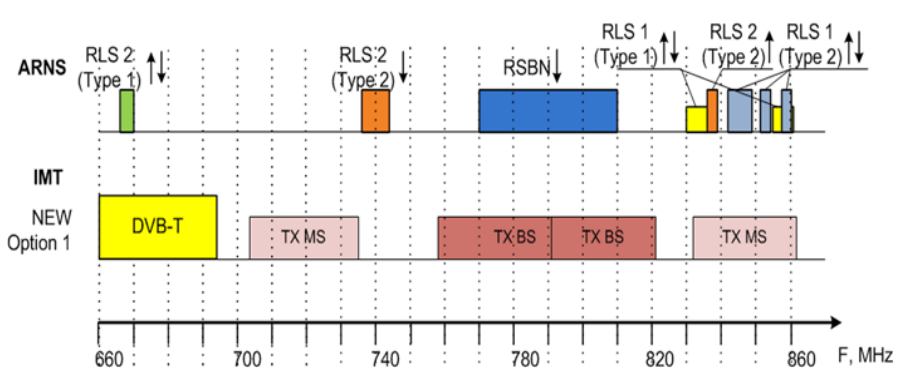

Fig. 1. ARNS and possible MS frequency utilization.

As shown in the Table I systems (systems' segments) operating in the $694 \mathrm{MHz}-790 \mathrm{MHz}$ frequency band operate in "air-to-ground" direction.

Depending on the applied mobile service channel arrangement, different scenarios of interferences can occur. One possible channel arrangement is presented in Fig. 1.

If conventional duplex direction operating in FDD mode is chosen (i.e. base station transmits within the upper band and mobile terminal transmits within the lower band), the RSBN ground receivers will be interfered by base stations of mobile service. If implementation of such channel arrangement is chosen, then the RLS 2 receiver will likely be operating in a duplex gap of mobile service frequency arrangement applying the APT band plan; or in the uplink of the mobile service for a $2 \times 40 \mathrm{MHz}$ channel arrangement [2].

Mobile service (MS) base station and aeronautical radionavigation service (ARNS) station parameters used to derive the respective interference power threshold and required path loss are summarized in Table $2[2,11,12]$.

TABLE II

BASIC CHARACTERISTICS OF THE MS AND ARNS SYSTEMS IN THE $694 \mathrm{MHz}-790 \mathrm{MHZ}$ FREQUENCY BAND

\begin{tabular}{|l|l|l|l|l|}
\hline Parameter & $\begin{array}{l}\text { MS } \\
\text { base } \\
\text { station }\end{array}$ & $\begin{array}{l}\text { MS } \\
\text { mobile } \\
\text { station }\end{array}$ & $\begin{array}{l}\text { RSBN } \\
\text { ground } \\
\text { receiver }\end{array}$ & $\begin{array}{l}\text { RSBN } \\
\text { aircraft } \\
\text { transmitter }\end{array}$ \\
\hline Frequency, MHz & 780 & 740 & 780 & 780 \\
\hline $\begin{array}{l}\text { Transmitter } \\
\text { power } \\
\text { (maximum), } \\
\text { dBm }\end{array}$ & 36 & 16 & - & 62 \\
\hline $\begin{array}{l}\text { Rx noise figure, } \\
\text { dB }\end{array}$ & 5 & 9 & 5 & - \\
\hline $\begin{array}{l}\text { Rx antenna gain } \\
\text { (incl. feeder } \\
\text { loss), dBi }\end{array}$ & 12 & -3 & 22 & - \\
\hline $\begin{array}{l}\text { Tx antenna gain } \\
\text { (incl. feeder } \\
\text { loss), dBi }\end{array}$ & 12 & -3 & - & 3.5 \\
\hline $\begin{array}{l}\text { Antenna height, } \\
\text { m }\end{array}$ & 30 & 1.5 & 10 & 3000 \\
\hline $\begin{array}{l}\text { Antenna tilt, } \\
\text { degree }\end{array}$ & 3 & - & - & - \\
\hline $\begin{array}{l}\text { Channel } \\
\text { bandwidth, MHz }\end{array}$ & 5 & 5 & 3 & 3 \\
\hline
\end{tabular}

The RSBN system is the non-directional, two way radionavigation aids capable to determine the azimuth and distance of the aircraft from the point where RSBN is installed.

\section{A. RSBN ground station receiver's antenna characteristics}

It shall be noted that typical area control and air surveillance radars uses the cosecant square pattern antenna or a fan beam antenna [10]. Both types give desirable characteristic, in terms of horizontal and vertical radiation pattern.

Fig. 2 shows the possible antenna diagram of the aeronautical radio-navigation services ARNS (the radar range dependence on the aims of height). This antenna pattern could be considered as average of the radars systems ( $\mathrm{x}$-axis up to few hundred kilometers, y-axis vertical pattern from $0^{\circ}$ to $90^{\circ}$ degrees). The maximum operating range of the ARNS can be calculated using the so-called radar equation. This distance is taken into account as a theoretical range (the real distance depends on the particular area relief). [16]:

$$
R=\sqrt[4]{\frac{P_{S} \cdot G^{2} \cdot \lambda^{2} \cdot \sigma}{P_{E} \cdot(4 \pi)^{2}}},
$$

where:

$P_{S}-$ maximum transmitted power, $\mathrm{W}$;

$P_{E}-$ received power, $\mathrm{W}$;

$G$ - maximum antenna gain, $\mathrm{dBi}$;

$\lambda$ - wavelength, $\mathrm{m}$;

$\sigma-$ cross section of the antenna, $\mathrm{m}^{2}$;

The ARNS parameters like maximum transmitted power, maximum antenna gain and wavelength can be considered as the constants because most of the radars have mostly the same parameters or they are balanced within a very small interval. The cross section of the antenna is miscellaneous (in this calculation were used $1 \mathrm{~m}^{2}$ ). The sensitivity level of the radar was as $P_{\text {Emin }}[16]$.

The antenna patterns according this initial data were created in SEAMCAT.

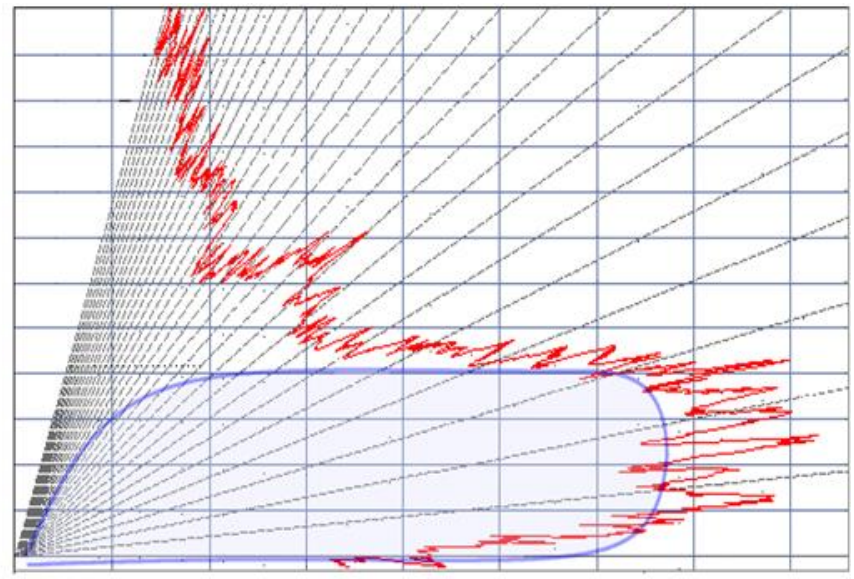

Fig. 2. Cosecant squared antenna pattern [10]. 


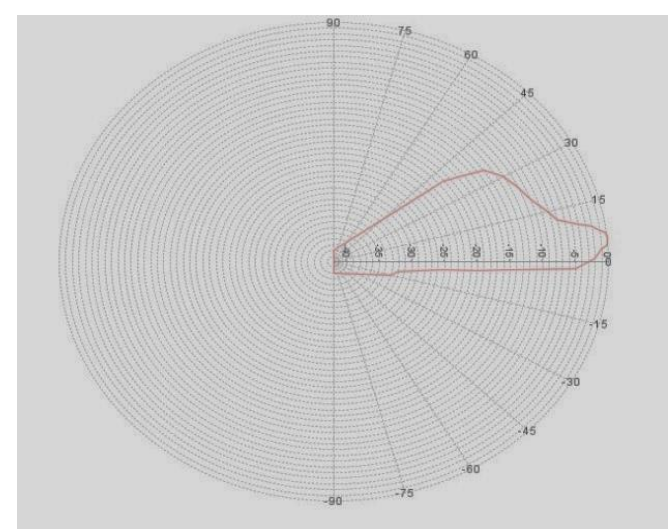

Fig. 3. Cosecant square antenna pattern used in SEAMCAT (vertical pattern).

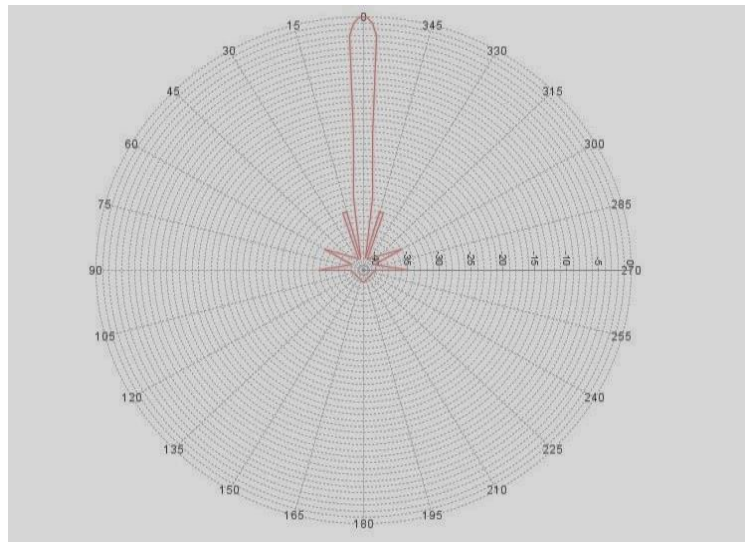

Fig. 4. Cosecant squared antenna pattern used in SEAMCAT (horizontal pattern).

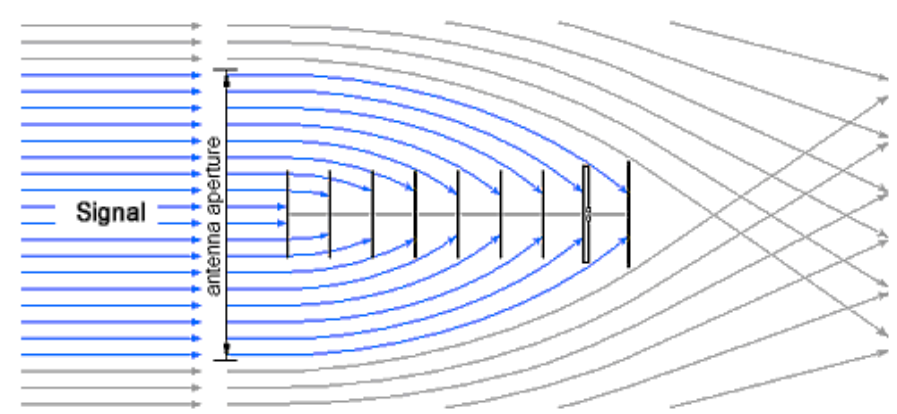

Fig. 5. Antenna aperture.

Fig. 2 and 3 show the possible antenna patterns for the area control and air surveillance radars. Those patterns are similar like in ECC Report 174 (See Fig. 13 and 14) [15].

The power density at the receiving antenna $S_{e}$ and the aperture of the antenna $A_{W}$ are two main parameters which describe received power PE of the ARNS systems:

$$
P_{E}=S_{b} \cdot A_{w}
$$

where:

$S_{e}$ - power density at receiving place;

$A_{W}-$ aperture of the antenna, $\mathrm{m}^{2}$.

The aperture qualifies efficiency coefficient $K a$ of the particular antenna. This parameter in average varies from 0.6 to 0.7 . [16].
The aperture of antenna is related with geometrical area of the antenna:

$$
A_{w}=A \cdot K_{a},
$$

where:

$A$ - geometric antenna area, $\mathrm{m}^{2}$;

$K_{a}$ - antenna efficiency.

Effective aperture (See Fig. 5) will be a useful concept for calculating received power from a plane wave.

It is possible to show formula (2) in more detailed form. The power received $P_{E}$ is then calculated:

$$
\left\{\begin{array}{l}
P_{E}=S_{b} \cdot A_{w} \\
A_{w}=A \cdot K_{a} \\
S_{b}=\frac{P_{r}}{4 \pi \cdot R_{2}^{2}}
\end{array}\right\} \Rightarrow P_{E}=\frac{P_{r}}{4 \pi \cdot R_{2}^{2}} \cdot A \cdot K_{a},
$$

where:

$P_{r}-$ reflected power, $\mathrm{W}$;

$R_{2}-$ distance between object and antenna, m.

The distances radar-target $R_{1}$ and target-radar $R_{2}$ are often not equal due to different relief and possible reflections. So we have analysed these ranges separately [16]:

$$
\left\{\begin{array}{l}
P_{E}=\frac{P_{r}}{4 \pi \cdot R_{2}^{2}} \cdot A \cdot K_{a} \\
P_{r}=\frac{P_{S}}{4 \pi \cdot R_{1}^{2}} \\
R_{1}=R_{2}
\end{array}\right\} \Rightarrow P_{E}=\frac{P_{S} \cdot G \cdot \sigma}{4 \pi \cdot R^{4}} \cdot A \cdot K_{a}
$$

where:

$G$ - maximum antenna gain, $\mathrm{dBi}$.

Formula (5) shows received power of the radar. This equation can be adapted in SEAMCAT calculations: in perspective of received radar power - wanted and unwanted power.

\section{B. RSBN ground station receiver's filter characteristics}

RSBN ground station receiver's filter mask is based on Recommendation ITU-R M.1830. This Recommendation complements the technical characteristics and and protection criteria for ARNS systems operating in the $645 \mathrm{MHz}-862 \mathrm{MHz}$ frequency band for relations not covered by RRC-06 and can be used by concerned administrations as technical guidelines for bilateral discussion and for estimations of compatibility with other radiocommunication services of administrations not party to the RRC-06 Agreement [14]. 


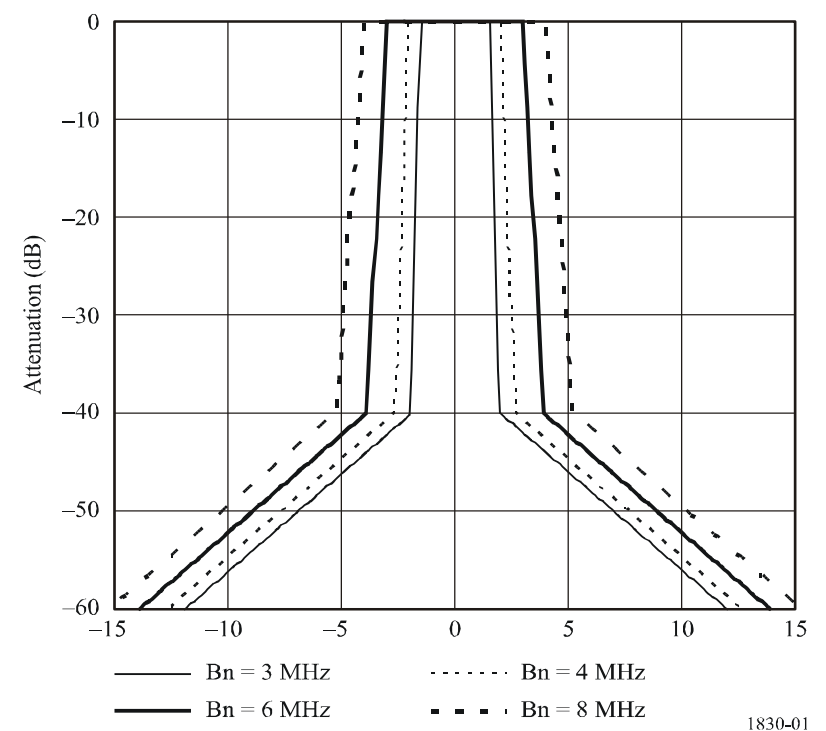

Fig. 6. Perspective ARNS receiver filter mask for the $645 \mathrm{MHz}-862 \mathrm{MHz}$ band [9].

In accordance with RR No. 5.312, in the $645 \mathrm{MHz}-862 \mathrm{MHz}$ frequency band also could be used ARNS systems on the primary basis: newly formed IMT networks must not create additional interference. The main types of radionavigation systems are listed below [9]:

$>\mathrm{RSBN}$ - radio systems for short-range navigation ;

$>$ ATC secondary radars - duplex systems for the air traffic control which consists of the two stations - ground radar and the airborne transponder;

$>$ ATC primary radars - ATC systems for aerodrome and route area control.

RSBN ground receiver's filter mask is shown in Fig. 6. The masks are based on theoretically obtainable capabilities in modernization of ARNS receiving filters and actually they refer to practically ideal characteristics of filtration (rectangularity factor of at least 1.3 at $-40 \mathrm{~dB}$ ). [9]

\section{THEORETICAL ANALYSIS}

There are different types of triggers such as coordination distances, aggregated field strength triggers and single field strength triggers. Each of them has both benefits and drawbacks.

Coordination distances: the choice of a certain distance might be seen as a simple and practical way to decide on when more detailed coordination is needed. However as the agreed distance has to be based on certain scenario, it might not be the most spectrum efficient and flexible method to use. The divergence in deployment from studied scenario will create differences in the sharing situation between the two services.

Aggregated field strength trigger: Independent of the deployment scenario or the technology used, it will provide in most cases the right amount of protection. At the stage of identifying the possible affected administrations this trigger value can be successfully used.

Single field strength trigger: it may be seen as a combination of the methods above. Nevertheless it has to be based on a certain scenario but instead of a coordination distance a field strength trigger for each station is defined. Such trigger will also have the ability to protect the concerned service if the technology used is changed. However the single field strength trigger may have some disadvantages as the coordination distance trigger since it is based on a certain scenario.

Trigger based on Interference-to-Noise ratio $(I / N)$ may be seen as the way of protecting a specific service, independent of the deployment scenario or the technology used.

For the protection of Mobile Service and evaluating the interference to ARNS caused by Mobile Service the $I / N=-6$ $\mathrm{dB}$ is used.

The theoretical calculations are performed using Minimum Coupling Loss (MCL).

According to Recommendation ITU-R V.573, the thermal noise threshold of a receiver can be determined by:

$$
P_{n}=10 \log \left(k T_{0}\right)+10 \log \left(10^{\frac{N F}{10}}-1\right)+10 \log B ;
$$

where:

$k$ - Boltzmann's constant $\left(1.3806488 \times 10^{-23} \mathrm{~J} \mathrm{~K}^{-1}\right)$;

$T_{0}$ - reference temperature of the receiver $(290 \mathrm{~K})$;

$N F$ - receiver noise figure $(\mathrm{dB})$;

$B$ - noise equivalent bandwidth of receiver $(\mathrm{Hz})$.

The thermal noise power for the receiver of $1 \mathrm{MHz}$ bandwidth:

$$
P_{n}=-114+10 \log \left(10^{\frac{N F}{10}}-1\right) ;
$$

Path loss or isolation between MS base station and ARNS ground stations using $I / N=-6 \mathrm{~dB}$.

The interference level at the ARNS ground station due to the mobile service is given by:

$$
P_{I_{-} \text {ARNS }}=-105.8 \mathrm{dBm} / 3 \mathrm{MHz}+(-6 \mathrm{~dB})=-11.8 \mathrm{dBm} ; \quad(8)
$$

The total maximum e.i.r.p. (equivalent isotropically radiated power) of base station transmitter is given by:

$$
P_{\text {EIRP_BS }}=36 \mathrm{dBm}+12 \mathrm{dBi}=48 \mathrm{dBm} \text {; }
$$

Then to ensure that there is no interference between interferer and victim the isolation required is given by:

$$
\text { Isolation }=P_{E I R P \_B S}+G_{R X}-P_{I_{-} A R N S}
$$

where:

$G_{R x}$ - the receiver antenna gain including cable losses.

The isolation is then converted to separation distance using the propagation model from Recommendation ITU-R P.1546-4 for path loss calculation (for $10 \%$ of time and $50 \%$ of location). This radio wave propagation model is developed for point-to-area path loss predictions for network planning of the broadcasting, land mobile and certain fixed services operating in the frequency range $30 \mathrm{MHz}$ to $3000 \mathrm{MHz}$ and is suitable for the distance range $1 \mathrm{~km}$ to $1000 \mathrm{~km}$. Isolation required for the MS base station transmitter and ARNS receivers are given by:

$$
\text { Isolation }_{B S \_A R N S}=186.6 \mathrm{~dB} \text {; }
$$

The required protection distances between ARNS stations and the MS base stations are $132 \mathrm{~km}$. This protection distance will be verified in SEAMCAT simulations. 


\section{INTERFERENCE SCENARIO}

\section{A. SEAMCAT simulation tool}

SEAMCAT - spectrum engineering advanced Monte Carlo analysis tool. Monte Carlo method is widely used for the simulation of random processes. The principle of this method is to take samples of random variables from their defined probability density functions. In SEAMCAT environment these functions are called as "distributions". Hence, the first step is to define the distribution of possible values of the parameters of radiocommunications system under study (e.g. operating frequencies, powers, antenna heights, positions of transmitter and receiver, etc.). Then the toll analysis uses these distributions to generate random samples (they are called snapshots or trials in the program). In the next step SEAMCAT calculates the strength of the desired signal and for interfering signal for each trial. The results are stored in data arrays. Then SEAMCAT in each snapshot compares interference criterion of the wanted and unwanted signals at victim receiver. In the last step the tool gives the probability of interference [13].

In SEAMCAT software tool we always have to describe (Fig. 7):

$>$ Interfering System Link with Interfering Link Transmitter and Interfering Link Receiver.

> Victim System Link with Victim Link Transmitter and Victim Link Receiver.

The result of the Interference Link are calculated at each snapshot with the possible interference criterion - carrier to interference ratio $\mathrm{C} / \mathrm{I}$, carrier to interference and noise ratio $\mathrm{C} /(\mathrm{I}+\mathrm{N})$, interference to noise ratio $\mathrm{I} / \mathrm{N}$, noise and interference to noise ratio $(\mathrm{N}+\mathrm{I}) / \mathrm{N}$ and possible propagation models - Free Space model, Extended Hata, Extended Hata-SRD, ITU-R P.1546, Spherical diffraction, Longley Rice propagation, IEEE 802.11 Model C, Winner propagation. The list of propagation models are continually extended according to the requirements.

Using SEAMCAT such spectrum engineering cases could be addressed [13]:

$>$ Generic co-existence (sharing and compatibility) studies between different radiocommunications systems (mobile, broadcasting, fixed) operating in the co-channel or adjacent channel case;

$>$ Evaluation of masks for transmitter or receiver;

$>$ Evaluation of various limits for given system parameters, such as unwanted emissions in a spurious domain as well as out-of-band emissions, blocking and intermodulation levels.

With SEAMCAT various radiocommunications services could be modelled, such as [13]:

$>$ Broadcasting Services - terrestrial systems and Earth stations;

> Mobile Services - Land Mobile Systems (LMS), Short Range Devices (SRD) and components of satellite systems based on Earth surface;
Fixed Services - Point-to-Point (P-P) and Point-toMultipoint (PMP)_fixed systems.

SEAMCAT tool is very popular in European Union Spectrum Engineering working groups.

\section{B. Interference scenario implementation to SEAMCAT}

The idea of interference scenario is shown in Fig. 8: Regardless the channel arrangement and duplex type used in mobile service, the base station to ARNS ground receiver interference scenario corresponds to the worst coexistence case.

The LTE average cell radius was assumed to be up to $8 \mathrm{~km}$. During the simulations small LTE network was created (shown in Fig. 9): each LTE BS has three sectors with one LTE user which is occupied $5 \mathrm{MHz}$ bandwidth channel. LTE BS and LTE UE emission masks are based on ETSI TS 136104 V10.2.0, ETSI TS 136101 V10.6.0 respectively.

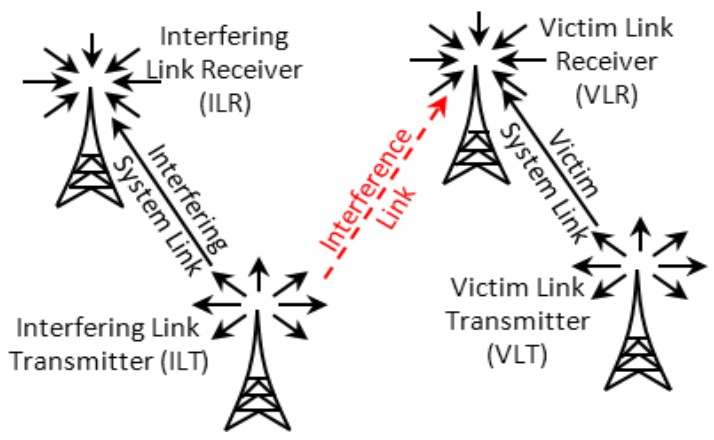

Fig. 7. SEAMCAT simulation area [13].

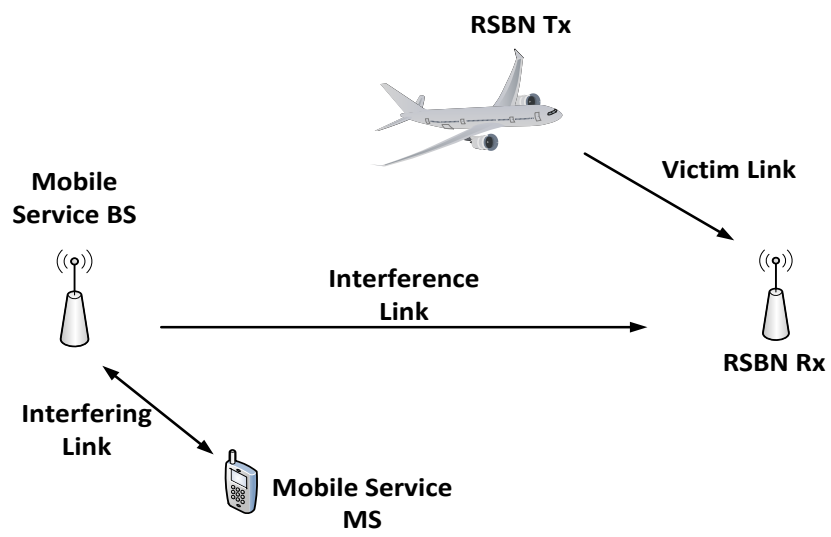

Fig. 8. Simulation scenario: Mobile Service BS interferes ARNS.

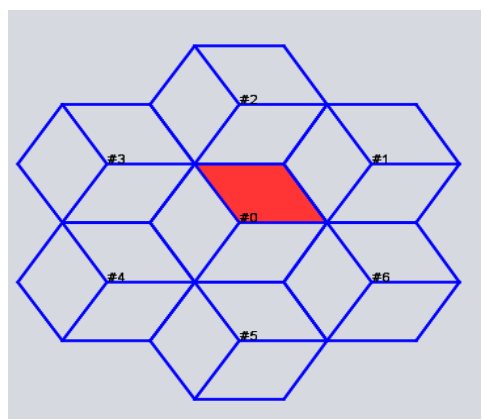

Fig. 9. LTE network. 


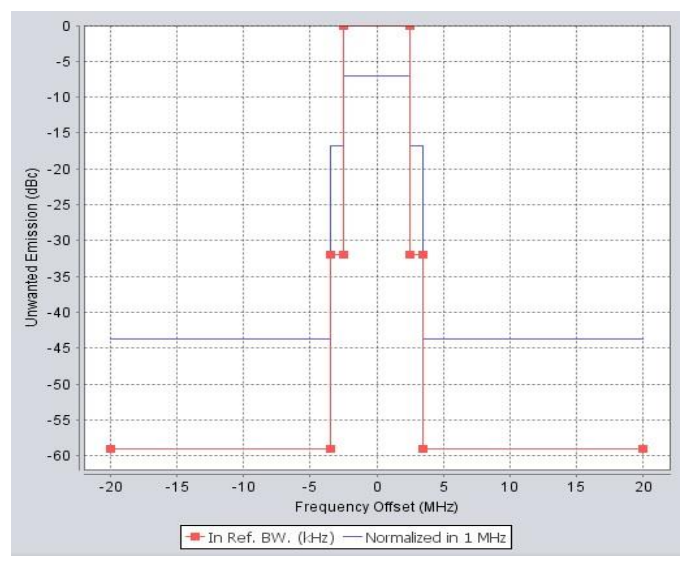

Fig. 10. LTE BS emission mask based on ETSI TS 136104 V10.2.0.

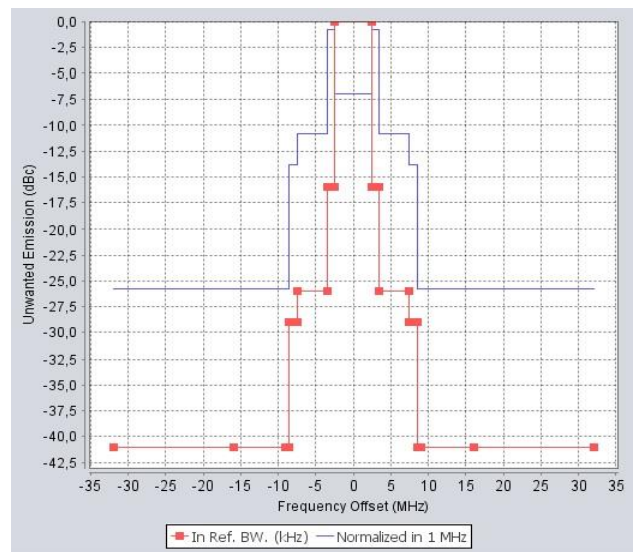

Fig. 11. LTE UE emission mask based on ETSI TS 136101 V10.6.0.

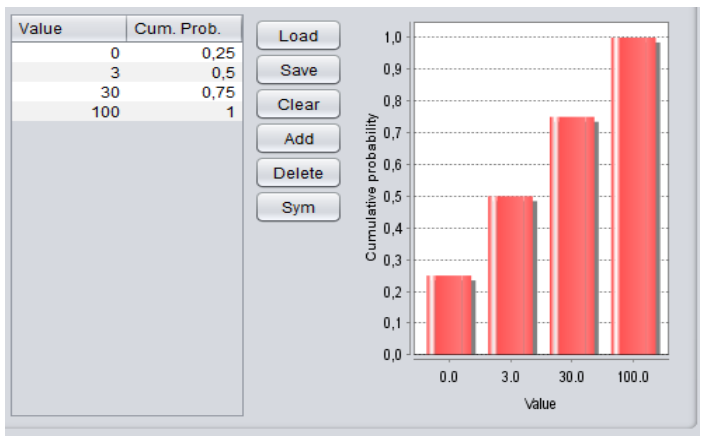

Fig. 12. MS mobile station's mobility.

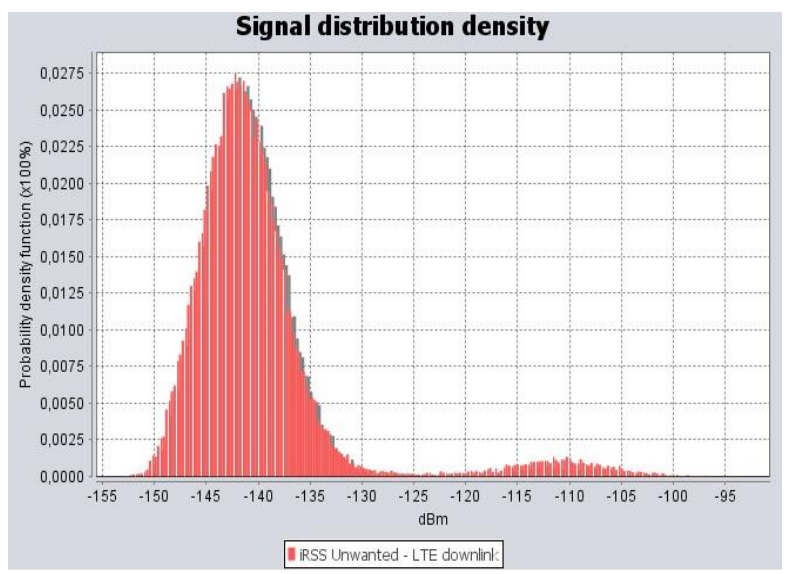

Fig. 13. Interference signal distribution.
This particular interference analysis is co-channel, but it was used LTE BS emission mask as seen in Fig. 10 to analyse real life situation. In this case out-of-band emission has small influence to RSBN receiver.

Fig. 11 shows possible LTE mobile station's out-of-band emission. LTE UE emission mask was created to reflect real life situation. In this interference analysis LTE UE's out-ofband levels can have influence in low distance simulations.

SEAMCAT can describe the MS mobile station mobility $(\mathrm{km} / \mathrm{h})$.

For simplicity as seen in Fig. 12 it could be separated into four different groups with uniform probability: $0 \mathrm{~km} / \mathrm{h}$ corresponds as to no movement, $3 \mathrm{~km} / \mathrm{h}$ corresponds to walking, $30 \mathrm{~km} / \mathrm{h}$ corresponds to urban driving condition and $100 \mathrm{~km} / \mathrm{h}$ corresponds to motorway driving.

ARNS receiver always operating at noise level: $-110.6 \mathrm{dBm} / 1 \mathrm{MHz}$ (or $-105.8 \mathrm{dBm} / 3 \mathrm{MHz}$ ). Typical distance between ARNS $T x$ and $R x$ is up to $600 \mathrm{~km}-800 \mathrm{~km}$. During the simulations in SEAMCAT $600 \mathrm{~km}$ separation distance was chosen.

\section{V.MONTE-CARLO SIMULATION RESULTS}

The main aim of this sector is to verify the obtained results in analytical calculations with results in SEAMCAT calculations. In each case there were 20000 calculations. The ITU-R P.1546 propagation model with for $10 \%$ of time and $50 \%$ of location for unwanted signal and for $50 \%$ of time and $50 \%$ of location for wanted signal was used. The propagation model in SEAMCAT was simplified - radio wave propagation only over the land paths, no antenna heights with negative values and only flat terrain model were used.

The required protection distances between ARNS stations and the MS base stations according to analytical calculations are $132 \mathrm{~km}$.

Note $^{1}$ : interference probability less than $5 \%$ is considered like a sufficient level.

The obtained results are showing that electromagnetic compatibility between LTE BS and ARNS $R x$ is possible at separation distances above $100 \mathrm{~km}$.

It is possible to analyse the interference signal probability at the RSBN receiver point:

Fig. 13 shows the interference signal distribution at the RSBN receiver point as the separation distance between MS base station and RSBN ground receiver is $130 \mathrm{~km}$. The signal varies from $-101 \mathrm{dBm}$ to $-150 \mathrm{dBm}$, average signal level is 140. It is seen that the majority of interference signal values are in the interval between $-130 \mathrm{dBm}$ and $-150 \mathrm{dBm}$.

TABLE III

MONTE-CARLO SIMULATION RESULTS - MS BASE STATION INTERFERES RSBN GROUND RECEIVER

\begin{tabular}{|l|l|}
\hline $\begin{array}{l}\text { Separation distance between MS } \\
\text { base station and RSBN ground } \\
\text { receiver, } \mathbf{k m}\end{array}$ & Interference probability ${ }^{\mathbf{1}} \mathbf{\%}$ \\
\hline 70 & 7.97 \\
\hline 100 & 4.82 \\
\hline 130 & 3.43 \\
\hline
\end{tabular}




\section{CONCLUSIONS}

This paper proposed an electromagnetic compatibility study in $694 \mathrm{MHz}-790 \mathrm{MHz}$ frequency band where Mobile Services and ARNS services will have to operate in parallel. This problem is currently of top importance as ITU and CEPT organizations by 2015 need to decide whether Mobile Service can operate within this frequency range.

Analytical calculations showed that the required protection distances between ARNS stations and the MS base stations are $132 \mathrm{~km}$. The obtained results from SEAMCAT simulations showed that separation distance should be above $100 \mathrm{~km}$.

In all the calculations $I / N=-6 \mathrm{~dB}$ interference criterion was used. The question is whether this criterion is not too strict. For example, the protection of ARNS against DVB-T is defined in the GE-06 Agreement which ensures the sufficient protection of ARNS: $I / N=\sim 30 \mathrm{~dB}$ according to the specific ARNS receiver parameter. For a balanced approach, similar protection against interference of mobile service should be considered.

\section{REFERENCES}

[1] RSPG Report on proposed spectrum coordination approach for broadcasting in the case of a reallocation of the $700 \mathrm{MHz}$ band, RADIO SPECTRUM POLICY GROUP, 2013

[2] Compatibility studies of the mobile service with the aeronautical radionavigation service in the frequency band $694-790 \mathrm{MHz}$ in Region 1, ITU Joint Task Group 4-5-6-7 working document, 2013 July.

[3] Generic case study on possible multiservice interference to ARNS, Document 4-5-6-7/359-E. 2013 October.

[4] A. Attar, V. Krishnamurthy, O.N. Gharehshiran "'Interference management using cognitive base-stations for UMTS LTE," IEEE Communications Magazine, Volume 49, Issue 8, IEEE, 2011, pp. 152-159.

[5] X. Hognian, S. Hakola "The investigation of power control schemes for a device-to-device communication integrated into OFDMA cellular system," in 2010 IEEE 21st International Symposium on Personal Indoor and Mobile Radio Communications, 2010, pp. 1775-1780.

[6] C.U. Castellanos et al. "Performance of Uplink Fractional Power Control in UTRAN LTE," in 2008 Vehicular Technology Conference, 2008, pp. 2517-2521.

[7] R. Love et al. "Downlink Control Channel Design for 3GPP LTE," in 2008 Wireless Communications and Networking Conference, 2008, pp. $813-818$.

[8] G. Boudreau "Interference coordination and cancellation for $4 \mathrm{G}$ networks,” IEEE Communications Magazine, Volume 47, Issue 4, IEEE, 2009, pp. 74-81.

[9] ITU-R Recommendation M.1830. Technical characteristics and protection criteria of aeronautical radionavigation service systems in the 645-862 MHz frequency band, ITU 2007.
[10] ITU-R Recommendation M.1851, Mathematical models for radiodetermination radar systems antenna patterns for use in interference analyses, ITU 2009.

[11] ETSI TS 136 101 V10.7.0. Evolved Universal Terrestrial Radio Access (E-UTRA). User Equipment (UE) radio transmission and reception, (3GPP TS 36.101 version 10.7.0 Release 10), ETSI 2012.

[12] ETSI TS 136104 V10.2.0. Evolved Universal Terrestrial Radio Access (E-UTRA). Base Station (BS) radio transmission and reception. (3GPP TS 36.104 version 10.2.0 Release 10), ETSI 2011

[13] Report ITU-R SM.2028. Monte Carlo simulation methodology for the use in sharing and compatibility studies between different radio services or systems, ITU 2001.

[14] GE-06 Agreement. Geneva, 2006.

[15] ECC Report 174. Compatibility between the mobile service in the band $2500-2690 \mathrm{MHz}$ and the radiodetermination service in the band 2700-2900 MHz, 2012.

[16] H.R. Raemer. Radar Systems Principles, CRC Press, USA, 1996. [E-book] Available: Google books.

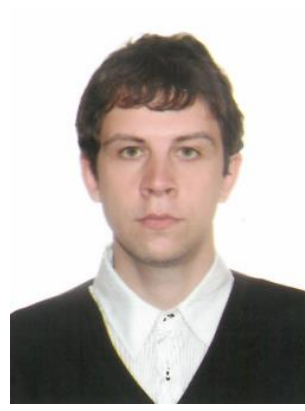

Evaldas Stankevičius received the $\mathrm{BSc}$ in telecommunications engineering from Vilnius Gediminas Technical University in 2010, and the MSc in 2012 respectively. He is currently continuing his researches in Vilnius Gediminas Technical University as a doctoral student in spectrum engineering field.

E. Stankevičius is the Chief Specialist of Spectrum Engineering division at Communications Regulatory Authority of the Republic of Lithuania for last three years.

His main research interests are in spectrum engineering problems in newly formed mobile network bands, radio propagation analysis, Electromagnetic compatibility studies in mobile, fixed and satellite services.

Evaldas is a student member of IEEE.

Postal Address: Naugarduko st. 41, Vilnius, Lithuania

E-mail: evaldas.stankevicius@rrt.lt

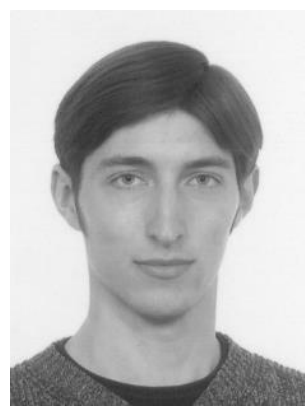

Šarūnas Oberauskas studied telecommunications physics and electronics at Vilnius University, Vilnius, Lithuania. He received the B. Sc. and M. Sc. degrees electric engineering in 2004 and 2006 respectively from Vilnius University.

S. Oberauskas is the Deputy Head of Spectrum Engineering division at Communications Regulatory Authority of the Republic of Lithuania for last fourth years.

His research interests include communications theory, 4G mobile broadband systems, electromagnetic field propagation theory and applications for communication systems.

Postal Address: Communications Regulatory Authority of the Republic of Lithuania, Vilnius, Lithuania, Algirdo str. 27A

E-mail: sarunas.oberauskas@ rrt.lt 